\title{
APPARATUS FOR MEASURING AND COUNTING MYELINATED NERVE FIBRES

\author{
BY
}

\author{
M. L. E. ESPIR* and D. T. C. HARDING \\ From the National Hospital for Nervous Diseases, Queen Square, and the Physics \\ Department, King's College Hospital
}

In recent years the fibre spectrum of myelinated nerves showing the proportion of fibres of different diameters has attracted increasing attention. For the purposes of some research requiring such fibre analysis, we developed apparatus for measuring and counting myelinated nerve fibres and it has proved to be of great assistance in this kind of investigation.

\section{Methods}

Transverse sections of nerve are prepared using a standardized histological technique, myelin sheaths being stained black with osmic acid, and photomicrographs of these sections are enlarged so that the final magnification is 1,000 times the original size. The apparatus described below is then used to measure and count all the myelinated fibres individually on the photographs; all measurements in millimetres represent microns in view of the selected magnification of 1,000 .

\section{The Apparatus}

Counters.-Fig. 1 shows the basic unit, which consists of a set of 15 electro-magnetic counters, each registering only when one particular size of fibre is measured. A sixteenth counter situated at the side of the instrument registers the total number of fibres of all sizes. An 18-way socket has been incorporated to take an appropriate plug through which all the leads from the measuring device (described later) are connected individually to the counters. Thus different types of measuring devices can be plugged in for use as required.

The electrical circuit of the counting unit, which is mains operated, is as in Fig. 2 (i.e., within the dotted line). Each individual counter is operated in a simple series circuit through the contact selector which is an integral part of the measuring device. The counter for indicating the total number of fibres measured is placed in series with the counter common line and this will register each time any individual counter operates. The counters used are the miniature post office type readily obtainable from government surplus suppliers, costing approximately $12 \mathrm{~s}$. $6 \mathrm{~d}$. each. The electrical components are quite standard costing in all about $£ 3$. The total cost, including all the component parts and materials used in the instrument, amounted to approximately $£ 15$.

*Present address: Leicester Royal Infirmary.
The instrument was built into a rexine covered case measuring 10 in. $\times 8 \frac{1}{2}$ in. $\times 5$ in.

Measuring Devices.-Three different types are described.

Protractor Type.-Fig. 3 shows a photograph of the perspex protractor. The graduated holes for measuring the diameters of the myelin sheaths have a size range from 2 to $16 \mathrm{~mm}$. Opposite each hole can be seen the brass contacts spaced in the form of an arc of smaller radius than the holes, each contact being connected back into the 18-way plug. The centres of the holes lie on an arc of $5 \mathrm{~cm}$. radius, and the contact selector arm, pivoted at the centre, is rotated to overlie the hole which has the same diameter as the myelin sheath being measured. The button is then depressed, and the hardened steel pin passes through the hole in the protractor to pierce the under-

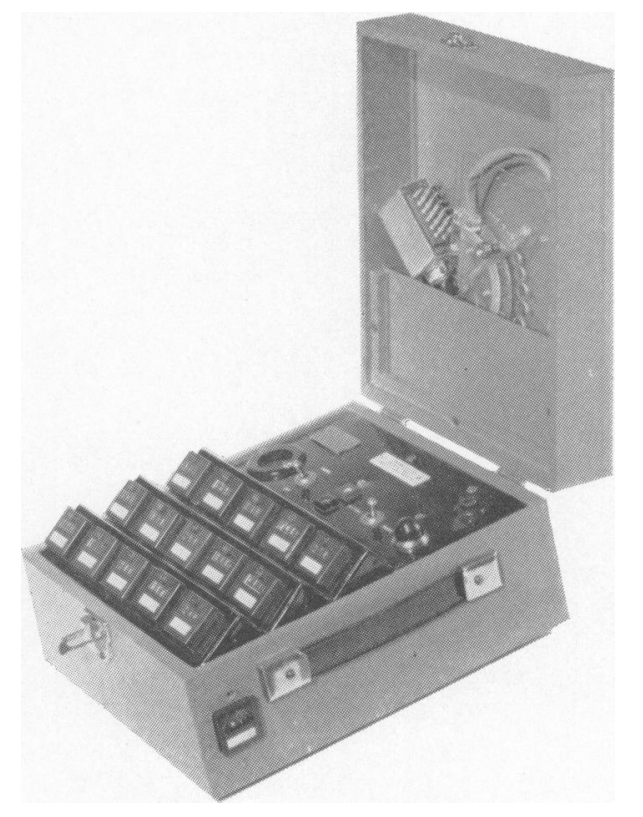

Fig. 1:-Universal counter unit showing 16 counters; the perspex protractor measuring device and the 18-way multiplug are housed in the case of the box. 

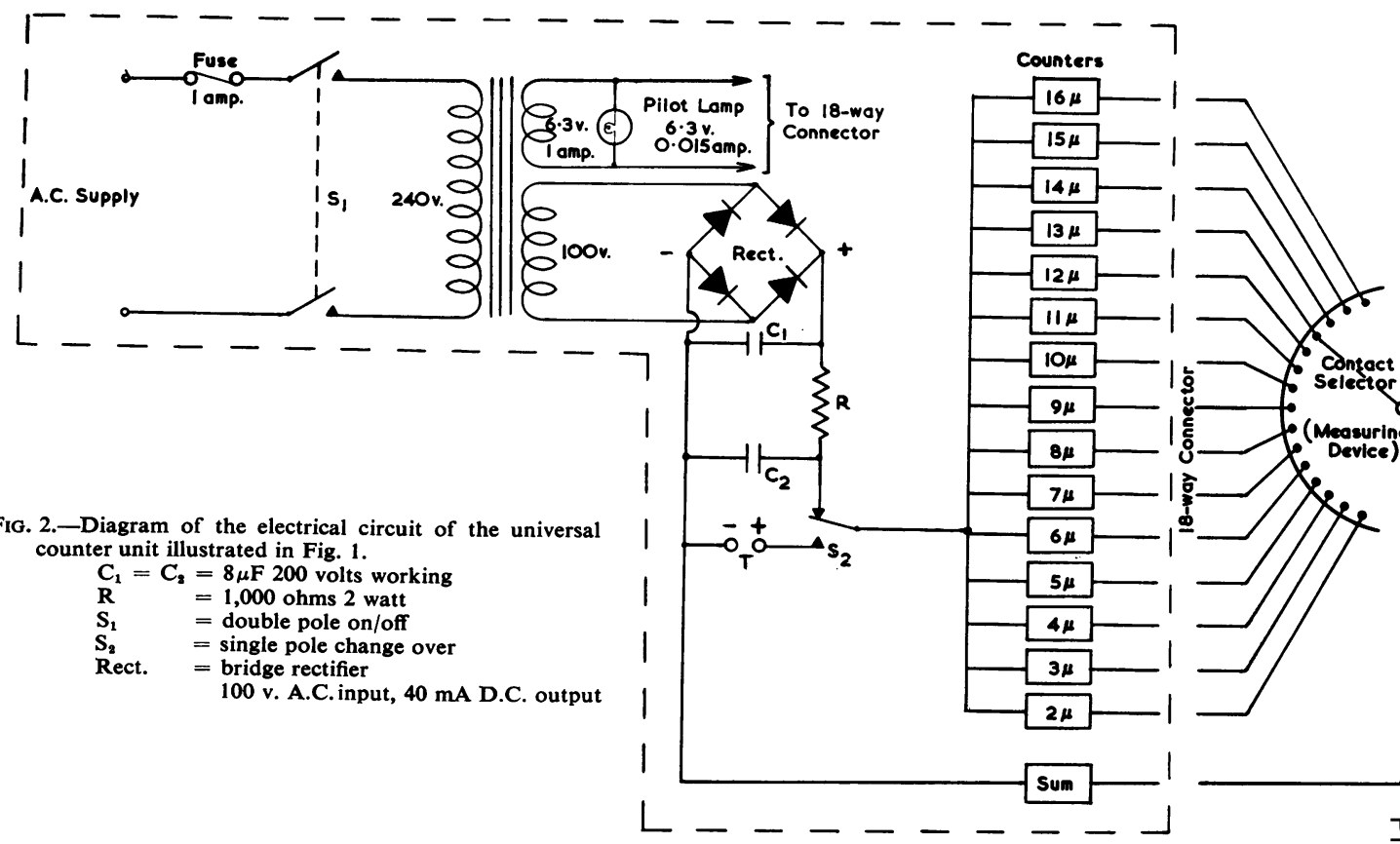

FIG. 2.-Diagram of the electrical circuit of the universa counter unit illustrated in Fig. 1.

$\begin{array}{ll}\mathbf{C}_{\mathbf{1}}=\mathbf{C}_{\mathbf{2}} & =8 \mu \mathrm{F} 200 \text { volts working } \\ \mathbf{R} & =1,000 \text { ohms } 2 \text { watt } \\ \mathbf{S}_{1} & =\text { double pole on/off } \\ \mathbf{S}_{2} & =\text { single pole change over } \\ \text { Rect. } & =\text { bridge rectifier }\end{array}$

100 v. A.C. input, 40 mA D.C. output

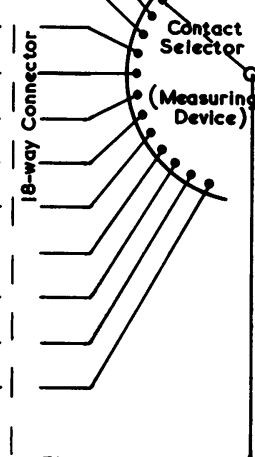

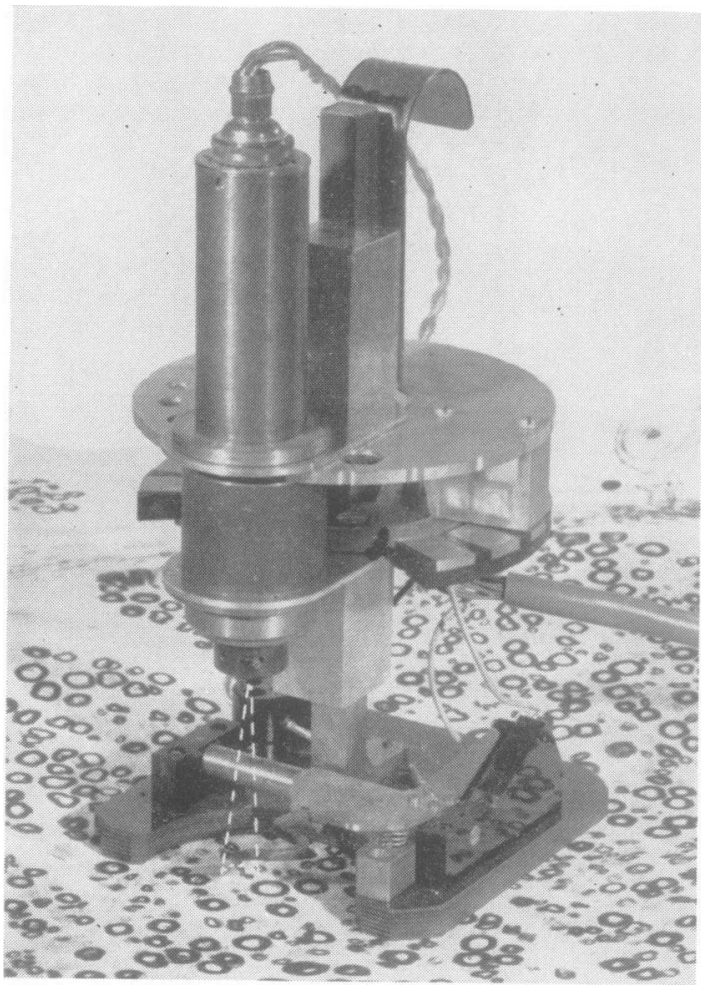

FIG. 4.-Light beam type of measuring device in use over the photomicrograph. The light beam is indicated by the dotted line.

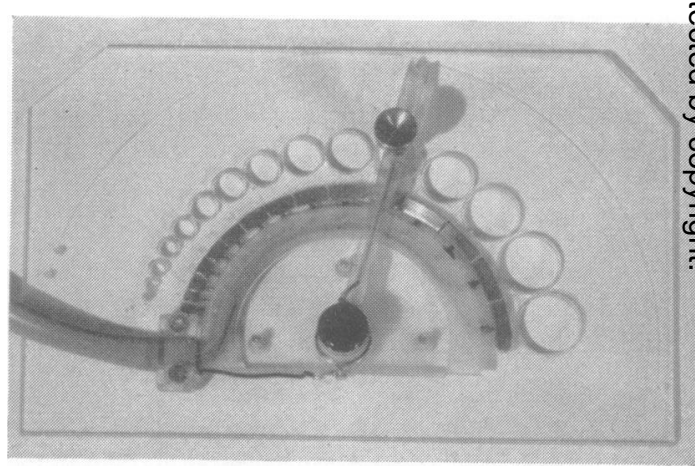

FIG. 3.-Perspex protractor type of measuring device.

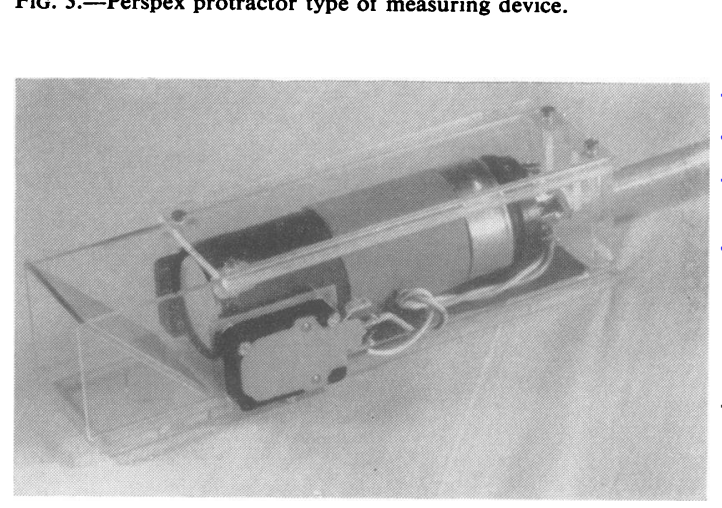

Fig. 5.-Cathode ray tube type of measuring device. 


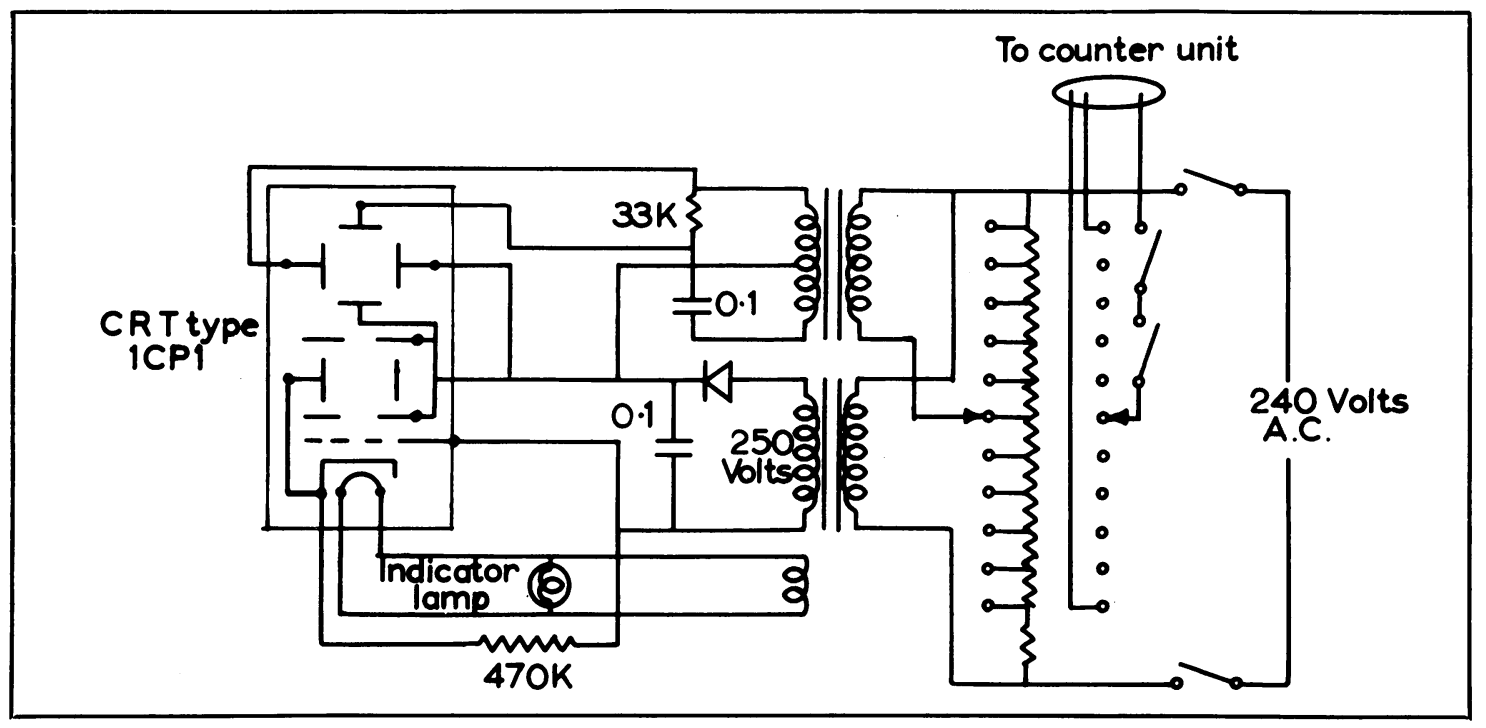

FIG. 6.-Diagram of the electric circuit of the cathode ray tube type of measuring device.

lying photograph, thus marking the centre of the nerve fibre measured. Simultaneously a metal brush on the underside of the sprung contact selector arm is made to touch the associated contact, completing the circuit back to the appropriate and 'total' counters. By releasing the button, a spring lifts the pin to its resting position, and the contact selector arm rises to its original position thus opening the electrical contact.

Light Beam Type.-A beam of light is shone from a fixed point above the photograph (Fig. 4), the 6.3 volts supply for the light source being obtained from the mains counter unit via two pins of the 18-way plug. A series of graduated holes cut in a circular metal plate can be rotated so that the beam of light is made to pass through each hole as desired. The diameter of the circle of light shining on the photograph can thus be altered from 3 to $16 \mathrm{~mm}$.

The instrument is moved over the photograph so that the centre of the beam corresponds with the centre of the nerve fibre to be measured, and the metal plate is rotated until the outline of the light fits the circumference of the myelin sheath. Separate contacts made for each hole in the metal plate are individually connected to the series of counters in the box through the multiplug. When the appropriate hole in the metal plate has been selected, contact is automatically made with the counter corresponding to the size being measured. The circuit is then completed for registration on the appropriate and total counters when the microswitch is triggered by depressing the lever. At the same time as the lever is depressed, the fibre measured is worked by the overlying point.

Cathode Ray Tube Type.-The electron beam of a 1 in. cathode ray tube is made to generate a circular trace on the fluorescent screen. An image of this illuminated ring is made to appear coincident with the nerve fibre being measured by viewing both the photomicrograph and the cathode ray tube screen through a half-silvered mirror set at $45^{\circ}$ (Fig. 5). Parallax is thus eliminated, giving less restriction on the angle of view. The diameter of the circular trace may be readily changed by adjusting the voltage applied to the deflecting circuit used, and the 11-position switch gives the range of 4 to 14 millimetres. An additional wafer on this switch selects the appropriate counter; Fig. 6 shows the electrical circuit.

In use, the diameter of the ring is matched to the myelin sheath being measured, and the perspex frame is then pressed to the paper. This causes the pin set in the base of the frame to pierce the centre of the fibre and at the same time operates the microswitch to register on the appropriate and total counters.

\section{Comment}

Using these measuring devices it is possible to analyse a nerve containing 1,000 fibres in approximately one hour, and, as with many machines, speed and accuracy can be increased with practice. In order to avoid the possible error of counting the same fibre more than once, each device is so constructed that a marker is linked with the switch which operates the counters, so that each fibre is clearly marked on the photograph at the same time as it is measured and counted. The resulting histogram is of interest for the study of peripheral nerves in health and disease, and a report of some of the findings will be published later.

Types 2 and 3 have the additional advantage that the beam or ring of light only has to be centred once over the fibre being measured, and can then 
be taken through the size range in the same position, i.e., without having to re-centre for each size being tested, as with the protractor type.

It is fully realized that there is still room for further improvement in the design of devices of this kind, and different methods of measuring other than the three described may be used. It is also possible that similar devices may have an application for other work involving counting and measuring, in which case the number (and shape) of gradations with corresponding counters may be altered according to the range required and purpose of the analysis. The multiplug is therefore invaluable because different measuring devices can be connected for use with the same box of counters.

\section{Summary}

A universal counter unit and three different measuring devices have been described as having special advantages for the analysis of myelinated fibres from photomicrographs of nerve sections.

We should like to thank Dr. E. S. Schuster, O.B.E. (Oxford) and Mr. H. B. Morton (Department of Applieder Electrophysiology, National Hospital, Queen Square) for the measuring devices Types 2 and 3 respectively, and for their help in the preparation of this paper. We areen also grateful to the principal physicist, King's College Hospital, for facilities and advice. Photographs of the devices were taken in the Photographic Departments at the National and King's College Hospitals.

M. L. E. E. wishes to acknowledge with thanks the facilities and financial help made available by $\mathrm{Mr} \cdot \overrightarrow{0}$ Harold Edwards, Director of the Department of Surgery, $\frac{\bar{O}}{-}$ King's College Hospital Medical School, the work of his technician, Mr. Colin Urwin, who prepared the histological section and photograph of the nerve shown, and $\mathrm{D}$ a research grant (which has covered some of the cost of materials) from the Institute of Neurology, The National Hospital for Nervous Disease, Queen Square, where this. work was started as clinical assistant to Dr. R. W. Gilliatt. 